\title{
Grandir en harmonie: Guide de l'utilisateur
}

Population Council

Follow this and additional works at: https://knowledgecommons.popcouncil.org/departments_sbsr-rh

Part of the Community Health and Preventive Medicine Commons, Demography, Population, and Ecology Commons, Family, Life Course, and Society Commons, Health Communication Commons, International Public Health Commons, and the Medicine and Health Commons How does access to this work benefit you? Let us know!

\section{Recommended Citation}

"Grandir en harmonie: Guide de l'utilisateur." Dakar: Population Council, 2007. 


\section{Sommaire}

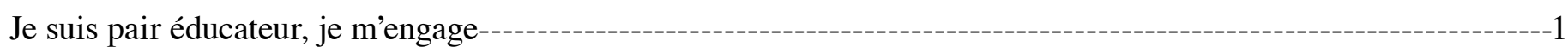

Qui est ma cible?---------------------------------------------------------------------------------------------------3

Je fais connaissance avec le curriculum "Grandir en Harmonie"-----

Je me familiarise avec la démarche du curriculum------

Comment j’organise les séances-----

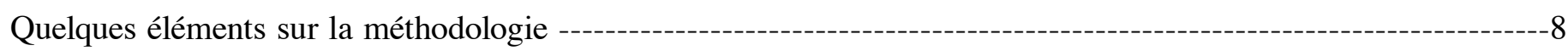

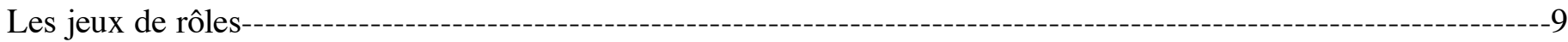




\section{Je suis pair éducateur, je m'engage}

\section{Qui suis-je?}

Je suis un(e) adolescent(e)/jeune âgé(e) entre 20 et 25 ans, choisi parmi mes pairs par la communauté. Je suis formé en Santé de la Reproduction des Adolescents et en communication interpersonnelle pour jouer efficacement le rôle d'éducateur de mes pairs. Chargé de mener des activités de Communication pour un Changement de Comportement (CCC) au niveau de ma communauté, je suis choisi selon les critères ci-après:

( Je suis adolescent(e)/jeune;

(1)ai envie de rendre service à ma communauté;

(.) Je suis membre d'une Organisation Communautaire de Base (OCB);

( Je suis accepté socialement et culturellement par ma communauté;

( ) Je suis favorable au programme de santé des adolescent(e)s/jeunes;

(2) Je suis désigné par mon Organisation Communautaire de Base (OCB);

() Je sers de modèle crédible pour le comportement social qu'on veut promouvoir;

() Je suis résident permanent dans la localité ;

(.) Je suis alphabétisé en français (sais lire et écrire le français);

O) Juis capable de communiquer clairement et de façon persuasive avec mes pairs;

(.) J'ai des capacités de leadership;

(1) J'ai le temps et l'énergie à consacrer à un travail bénévole;

(1) Je suis modeste, discret, disponible et bien apprécié par mes pairs;

() Je m'engage à mener des activités d'animation;

(.) A l'issue de ma formation, je suis mandaté par les responsables de la santé des adolescents pour soutenir le programme;

( Je travaille autour de la structure de santé de ma communauté.

\section{Quelle est ma mission? Quel est mon rôle?}

J'ai pour mission, sous la tutelle du responsable de la zone sanitaire, de contribuer à l'amélioration de la Santé des Adolescent(e)s/Jeunes (SR/Ado).

\section{Pour cela, je dois:}

( ) Eduquer, informer et sensibiliser les adolescent(e)s/jeunes dans le domaine de la SR/Ado à savoir: l'adolescence, les valeurs, la connaissance du corps, la sexualité, la grossesse, la prévention de la grossesse, les IST/VIH/SIDA et la parenté responsable. Ceci, pour les amener à adopter un comportement responsable face à leur santé et à leur développement;

() Orienter des adolescent(e)s qui en expriment le besoin, vers les structures de santé et les points d'information de ma zone de responsabilité;

(2) Assister pour l'accueil, le counseling et l'orientation vers le prestataire de santé;

( ) Planifier, exécuter, évaluer et suivre les activités de communication pour le changement de comportement (CCC);

(1) Faciliter la médiation en saisissant le comité technique local. 


\section{Quelles sont mes tâches?}

Pour bien jouer ces rôles et remplir ma mission, je dois m'acquitter correctement des tâches suivantes:

() Je planifie périodiquement, avec l'appui du comité technique, les activités de CCC/SR/Ado à exécuter:

$\Rightarrow$ Je mène des animations de groupes d'adolescents avec le curriculum "Grandir en Harmonie";

$\Rightarrow$ Je fais des entretiens (par des visites à domicile ou lors des rencontres fortuites);

$\Rightarrow$ Je mène des activités de masse (exemple: radios-crochets, feux de camp, podiums de sensibilisation, fora, conférences, etc.);

$\Rightarrow$ Je participe à l'organisation de journées d'information et de sensibilisation sur la SR/Ado des leaders administratifs, politiques, religieux, coutumiers et des populations cibles;

$\Rightarrow \mathrm{J}$ 'encourage la participation communautaire pour la résolution de problèmes SR/Ado;

$\Rightarrow$ Je participe aux activités d'information, de sensibilisation des parents;

$\Rightarrow$ Je mets à jour mes statistiques en remplissant régulièrement les outils de gestion;

$\Rightarrow$ Je fais des rapports d'activités mensuelles;

$\Rightarrow$ Je participe aux réunions de coordination des activités de SR/Ado;

$\Rightarrow$ Je participe à l'évaluation des activités menées;

$\Rightarrow$ J'appuie la structure de santé dans l'offre de services;

$\Rightarrow$ Je veille au bon entretien du matériel mis à ma disposition.

Au niveau des structures de santé, je suis chargé de faire du counseling aux adolescent(e)s/jeunes qui viennent en consultation. C'est-à-dire:

(1) Bien les accueillir en les saluant chaleureusement et en les mettant à l'aise et en confiance;

(1) M'entretenir avec eux pour m'encquérir de leurs problèmes/besoins et les aider;

(1) Les introduire auprès du prestataire au besoin;

(1) Aider le prestataire dans l'offre des produits et dans le suivi/relance des clients.

Le curriculum "Grandir en harmonie" est conçu pour m'aider à bien assumer mon rôle et à bien accomplir mes tâches.

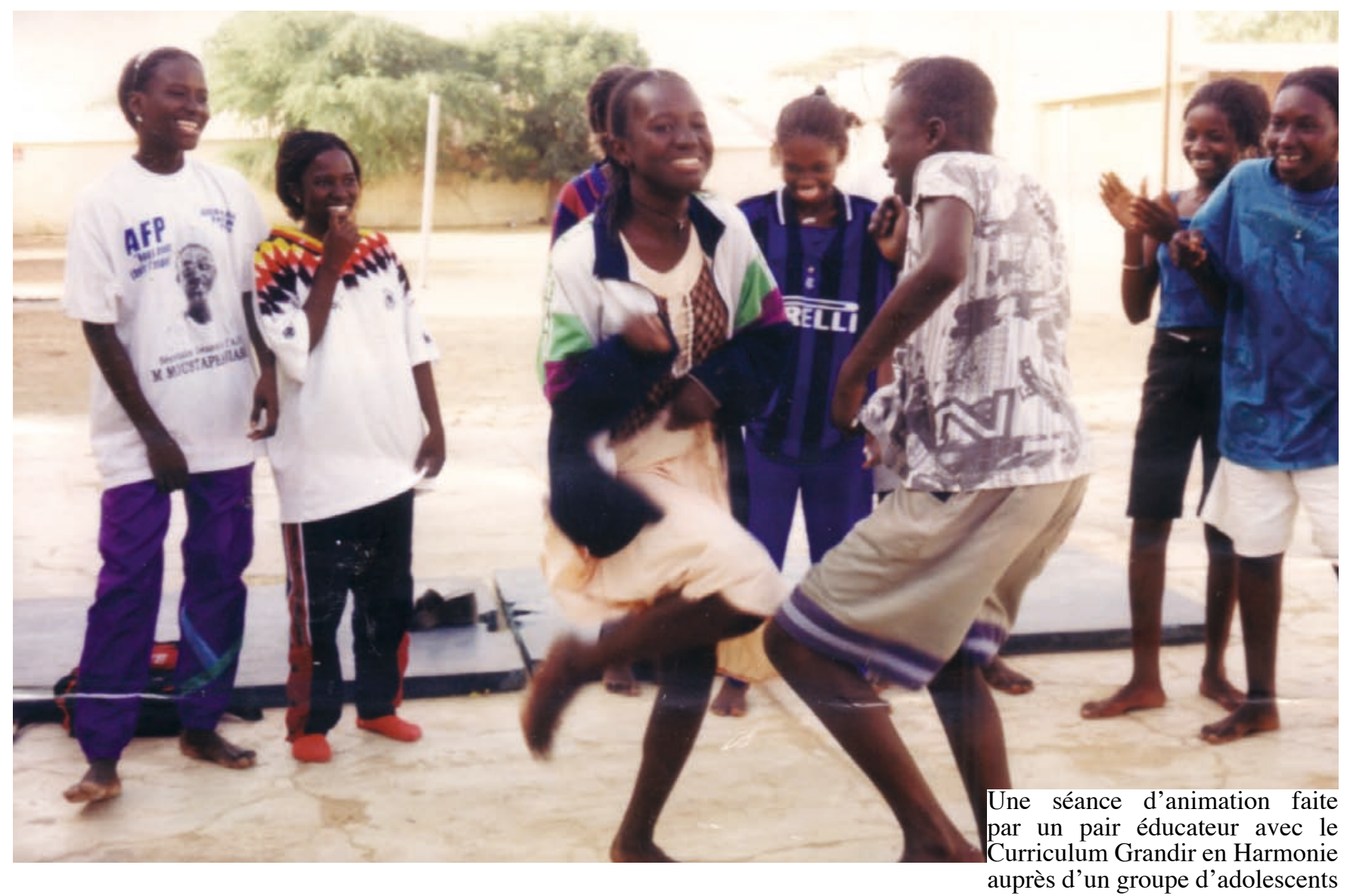




\section{Qui est ma cible?}

(1) Ma cible ce sont les adolescents âgés entre 10 et 19 ans et les jeunes jusqu'à 25 ans;

(1) Ma cible, garçon ou fille, vit une période des transformations physiques, psychiques, émotionnelles, et intellectuelles.

$\Rightarrow$ L'adolescent grandit, son organisme se modifie;

$\Rightarrow$ Les organes génitaux se développent;

$\Rightarrow$ Les caractères sexuels primaires apparaissent, c'est la puberté;

$\Rightarrow$ L'adolescent a du mal à savoir qui il est, ce qu'il veut;

$\Rightarrow$ Plus un enfant, pas encore un adulte, il a besoin de redéfinir ses goûts, ses forces, ses faiblesses, son identité;

$\Rightarrow$ Ma cible vit une période unique de la vie où on observe un cocktail d'émotions allant de la joie, l'excitation, l'anticipation, la sensibilité, à l'insécurité, l'irritabilité, l'impulsivité...

Ma cible est à la recherche d'un modèle.

L'adolescent a besoin d'être entouré, encadré, écouté et accompagné.

\section{Qui doit l'accompagner?}

(1) Les parents;

(1) Les enseignants;

(1) Les amis, les pairs;

(2) personnel de santé;

() Le personnel d'encadrement des jeunes.

\section{Pourquoi l'accompagner?}

Il peut choisir un mauvais modèle;

Il peut être tenté par l'usage de substances nocives (drogue, alcool, cigarette);

(1) Il veut être informé sur les changements physiques et émotionnels, sur la sexualité et a besoin de communiquer sur ces questions;

(1) Le manque de cadre de communication peut entraîner certains comportements à risque tels que la sexualité non protégée, les avortements provoqués, les IST/VIH/SIDA;

(1) Les adolescents éprouvent de la gêne à fréquenter les structures de santé, or les circuits parallèles et les réseaux informels auxquels ils peuvent avoir recours en cas de problèmes constituent des risques.

() Comment l'accompagner?

() Ne pas dire à l'adolescent(e) ou au jeune tout ce qu'il doit faire ou ce qu'il doit penser;

(1) L'accompagner dans ses propres efforts pour découvrir les différentes facettes de la vie et pour choisir sa propre voie;

(1) Etablir une relation de confiance.

Le pair éducateur devient un médiateur entre l'adolescent et les points de prestation de services adaptés à ses besoins. 


\section{Je fais connaissance avec le curriculum "Grandir en Harmonie"}

Grandir en harmonie propose des activités à faire avec les adolescents/jeunes et qui leur permettent de renforcer leurs capacités à prendre des décisions pour mieux se réaliser maintenant et dans le futur.

Grandir en harmonie est un curriculum d'animation en santé de la reproduction pour accompagner les adolescents dans leur transition vers l'âge adulte.

\section{Pour installer chez les adolescent(e)s/jeunes des compétences de vie, il est important:}

(1) De considérer le contenu de ce document comme un tout indivisible;

(1) De veiller à ce que tous les modules soient administrés au même groupe d'adolescents;

(1) D'avoir à l'esprit que la connaissance et l'estime de soi, l'empathie, la prise de bonne décision, l'aptitude à la communication, les relations interpersonnelles, la gestion des émotions et du stress, l'esprit critique et créatif, la résolution des problèmes, sont autant de compétences pour la vie qui demandent du temps pour être acquises.

\section{Grandir en Harmonie contient huit (8) modules:}

(.) Faisons connaissance;

(1) Les valeurs;

(1) Mon corps;

(1) Mes relations;

- Suis-je prêt(e) à avoir un enfant?

(2) Et pourtant je pouvais éviter la grossesse.

( IST/VIH/SIDA suis-je à risque?

() Plus tard je serai un parent responsable.

Pour réussir ma mission je prends soin avant chaque séance de revoir le contenu de la séance dans le document de références et une liste de questions/réponses les plus fréquentes. Ceci m'aidera à préparer les questions techniques que les participants poseront. Je n'oublie pas que les séances réussies sont celles qui sont bien préparées.

\section{Chaque session du curriculum contient:}

(.) Les objectifs;

() Une liste de matériels nécessaires (dépliants sur la Santé de la Reproduction, planches, cassettes, magnétophones, etc.);

(1) Ce que l'animateur/animatrice doit préparer avant de commencer;

(.) Le tableau synoptique du volume horaire de la session;

(1) Les étapes de la démarche et les instructions pour les dérouler;

(.) Une évaluation finale de chaque session;

() Les points à retenir. 


\section{Je me familiarise avec la démarche du curriculum}

Mon style d'animation: EIE: Exciter, Impliquer, Engager Je suis un facilitateur dans la mesure où je suis un Pair éducateur utilisant le modèle EIE.

A signifie que je dois Exciter les participants en utilisant la danse, le théâtre, les jeux, la musique et l'amusement. Le déroulement des sessions doit pousser les participants à avoir envie d'apprendre, pas à s'ennuyer. Les sessions doivent être éducatives et, en même temps, très divertissantes, d'où la notion d"'Education-Divertissement". Ainsi il me faut tout faire, à chaque étape, pour rendre les activités excitantes, attractives, exploratrices, originales...

T veut dire que j'Implique les participants dans tout le processus d'apprentissage, je les fais participer aux jeux de rôle, aux sketchs, à la réflexion, la recherche de solutions, la création de chansons, de danses, etc. Les participants sont au cœur du dispositif de formation.

$\mathrm{C}_{\text {proprifie que je dois encourager les participants à s'engager dans le processus. Ils sont maîtres de leur }}^{\text {signition. }}$ Pour que ce modèle soit efficace:

(1) J'encourage tous les membres de la séance d'animation à être des participants actifs;

O J'implique les participants dans tout le processus d'apprentissage;

- Je révèle les talents cachés des participants, leur connaissance et leur expérience.

\section{J'ai à l'esprit que je ne suis pas un formateur mais un facilitateur:}

(1) Un formateur fait passer l'information, je tire l'information à sa source;

(2) Un formateur prêche et dit aux élèves ce qu'ils doivent faire, je guide le processus de la prise de décisions;

(1) Un formateur exige le respect par la force et par d'autres moyens, je gagne le respect à partir de l'exemple;

(1) Un formateur impose ses valeurs aux élèves, j'aide les participants à trouver leurs propres valeurs.

Je facilite les sessions avec intégrité:

(1) J'utilise un langage adapté à ma cible;

(.) Je mène les sessions en respectant les règles que j'ai dégagées au début avec les participants;

Je suis ponctuel;

( Je suis prêt - la préparation des sessions doit être soigneusement planifiée à l'avance;

() Je donne l'exemple en rentrant moi même pleinement dans l'esprit de l'animation.

\section{Je motive et je change les participants}

Je suis sympa - je ne me fâche pas;

- Je casse la glace - je ne refroidis pas les participants;

Je comprends les participants - je ne porte pas de jugements de valeurs;

(O) Je partage le savoir - je ne prêche pas;

(1) J'tablis les règles de travail avec le groupe - je ne les impose pas;

O Je suis moi-même un modèle - je ne harcèle pas, je n'envoie pas de messages confus;

- Je suis motivé, et je motive les autres. 


\section{Comment j'organise les séances}

Je choisis et je compose mon groupe

Mon groupe doit avoir un maximum de 30 adolescent(e)s et être homogène.

A titre d'exemple les tranches d'âge comprennent les 10-12 ans, les 13-15 ans et les 16-19 ans. L'environnement dans lequel vit un adolescent peut le rendre mature ou pas. Par exemple: un jeune garçon de 11 ans, vivant dans un quartier populaire, livré à lui-même peut être déjà mature et sexuellement actif. Une jeune fille âgée de 13 ans, mariée et donc sexuellement active peut d'une certaine manière être plus mûre qu'une autre fille de son âge célibataire.

\section{Je dois donc faire attention en composant les groupes d'adolescent(e)s à leurs profils selon:}

(.) L'âge: (10-12 ans), (13-15 ans) et (16-19 ans);

(1) Le niveau d'instruction: instruits/non instruits;

L'exposition aux fléaux sociaux: à risques/pas à risques.

Le groupe sera aussi équilibré du point de vue du nombre de garçons et de filles.

Je m'entends avec le groupe sur un calendrier de rencontres selon la disponibilité de la cible

J'informe mon groupe que nous allons aborder toutes les sessions du curriculum. Ceci nécessite plusieurs rencontres.

Le curriculum peut être adapté à diverses situations. Le moment et la durée des séances peuvent être aménagés selon la disponibilité de la cible.

Je m'entends avec le groupe sur l'espace de rencontre

\section{Les critères suivants sont importants:}

(1) Lieu accessible aux jeunes;

() Lieu aéré;
(1) Espace vaste;

() A l'abri du bruit.

Le curriculum peut être déroulé partout, pourvu que le lieu convienne aux groupes d'adolescent(e)s/jeunes et qu'il soit accessible (dans les maisons, en plein air, au siége d'une association locale, lors des camps de vacances, etc....

Cependant, pour les thèmes sensibles, il est recommandé de les organiser dans des endroits assurant la discrétion et la confidentialité (Points de Prestation de Santé, points d'information, écoles, maisons de quartiers etc.)

L'espace disponible est un élément important pour réussir mon animation. Quand j'organise les jeux avec les listes de déclarations, il y a beaucoup de déplacements: il faut donc faire le maximum pour disposer de locaux où il y a assez d'espace pour que le groupe se sente à l'aise... Sinon, lorsque les lieux sont trop étroits, les jeux risquent d'être difficiles à organiser or, ils sont importants pour mettre une bonne ambiance et intéresser les adolescent(e)s/jeunes.

Si possible également, le lieu que je choisis sera aéré car, en période de chaleur, si on reste trop longtemps dans un milieu clos, l'attention des participants se dissipe au fil du temps... Si je choisis, avec le groupe d'adolescent(e)s/jeunes de faire l'animation en plein air, il faudra par contre veiller à ce que le lieu soit isolé du bruit et des passants... Il y a également des effets possibles sur l'utilisation de certains supports (magnétophone, tableau, etc.). Par exemple, trop de bruits alentour ou l'absence de prises de courant peuvent rendre impossible l'utilisation de supports qui étaient prévus lors d'une séance.

Cependant, par souci d'efficacité, je dois veiller à terminer toute session entamée avant de lever la séance. 
Je prépare le déroulement des séances.

Avant d'aborder chaque session, je dois bien lire la documentation y afférant contenue dans le document de références.

J'identifie et j'invite des personnes ressources si nécessaire.

Je m'assure de la disponibilité du matériel.

\section{Le matériel et les supports}

Je dispose d'un ensemble de matériels qui servent à faciliter les étapes du curriculum. Les principaux supports que je vais utiliser dans les différentes séances sont les suivants:

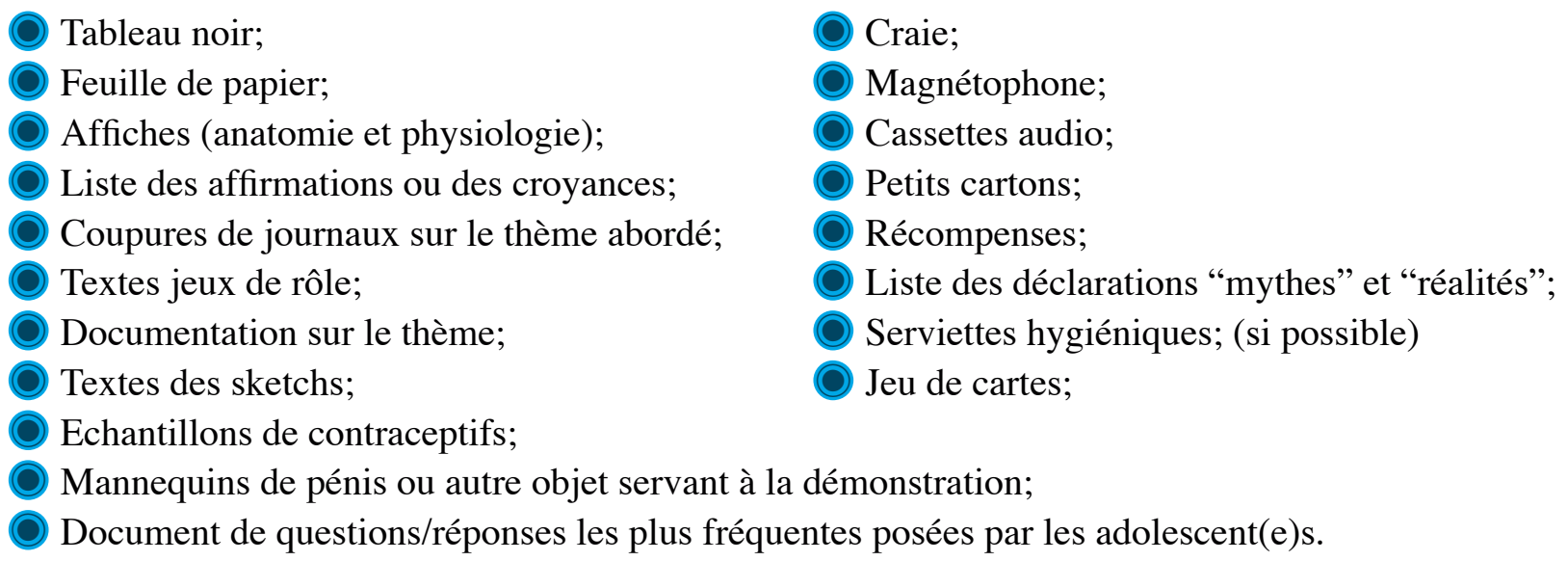

Je dois toujours veiller à une bonne préparation du matériel et des supports nécessaires à la séance choisie. Ils sont très importants pour une bonne animation du groupe, pour permettre un apprentissage actif et attrayant.

Je dois aussi être imaginatif et créatif pour penser à d'autres supports adaptés qui peuvent faciliter les discussions ou les apprentissages. En observant bien mon environnement, je peux toujours trouver des ressources utiles et moins chères (objets de récupération tels que bouchons, bouteilles vides, bouts de bois, bananes...).

Par ailleurs, je sais que les jeunes ne sont pas tous instruits, ma cible peut être analphabète, il me faut donc utiliser plus de couleurs, les dispositions dans l'espace (par exemple, à gauche pour “d'accord”, à droite pour "pas d'accord") etc....

\section{Je déroule ma session}

J'installe mon groupe confortablement en arc de cercle pour permettre l'interrelation entre l'animateur et le groupe.

J'explique à mon groupe que nous allons aborder aujourd'hui tel thème.

J'explique la méthodologie d'animation.

Je leur propose de créer un hymne qui leur soit propre, sur la base des mots-clés qui reflètent le contenu du curriculum Grandir en Harmonie, sa démarche, et son but.

Contenu: solidarité, confiance mutuelle, engagement, respect mutuel, amitié;

(1) Démarche: jeux, chants, discussions, échanges;

() But: connaissance de valeurs, connaissance de soi, maîtrise de soi, vie saine.

Après avoir déroulé tout le curriculum, le groupe pourra se trouver un slogan propre qui annonce un engagement à un changement de comportement.

Pour les groupes d'âge des 20-24 ans je peux aussi utiliser d'autres méthodologies tels que les panels, les causeries, les thé-débats... 


\section{Quelques éléments sur la méthodologie}

L'enchaînement et la complémentarité des différentes étapes d'une session (consignes à donner, exercices, réponses fréquentes, points à retenir, etc.) sont importants:

(1) La plupart des sessions durent entre quarante cinq minutes et une heure et demie;

(.) Certaines sessions sont plus longues que d'autres. Il me faut prendre mon temps et travailler au rythme des participants;

() Ce qui est important, c'est de les amener à comprendre les objectifs;

() Je dois m'arrêter et reprendre les points importants si les participants ne les comprennent pas;

O Je traite du renforcement du pouvoir, des capacités et compétences nécessaires pour la vie;

(.) La Communication pour un Changement de Comportement (CCC) nécessite du temps, de la pratique et de la patience;

() Pour m'aider dans mon travail de facilitation, je trouverais listées sous la rubrique Réponses fréquentes plusieurs propositions de réponses dont je pourrais me servir;

(1) Les réponses fréquentes sont des réponses types données par les personnes avec lesquelles je travaille (adolescent(e)s/jeunes du même âge);

( ) Si les participants sont bloqués, je peux leur suggérer quelques-unes des réponses fréquentes. Je peux aussi utiliser ces réponses fréquentes comme guides dans les séances de discussion. Elles ne représentent pas une liste de réponses correctes;

(1) Je n'hésite pas à recourir à des personnes ressources pour les questions pour lesquelles je n'ai pas de réponses.

Les exercices ont surtout pour but de faire participer les adolescent(e)s; ils permettent de les impliquer, de recueillir leur avis ou de les pousser à réfléchir sur des sujets donnés.

\section{Consignes particulières}

\section{Les discussions:}

() Je veille à une bonne distribution de la parole;

(1) Pour faciliter les changements de comportements, il est nécessaire de permettre aux différents acteurs de se rendre compte de la force des préjugés, des messages négatifs, qui les empêchent d'intégrer les valeurs et attitudes positives;

(1) La démarche qui consiste à donner des leçons aux participants n'est pas très efficace;

(2) Il faut que je les aide à expliciter leurs valeurs, à confronter leurs points de vue avec ceux des autres, etc...

(1) Pour tout cela, les discussions que l'on retrouve dans la plupart des étapes du curriculum sont très importantes;

(2) Dans l'organisation de ces discussions, je dois éviter de toujours mettre en avant ma propre position ou me baser sur mon propre point de vue pour affirmer qu'un tel ou un tel a raison...

() Mon rôle est surtout d'aider le groupe à discuter et, en m'appuyant sur les informations contenues dans le "document de références", aider chacun à se départir des préjugés, influences et messages négatifs qui l'empêchent d'avoir un comportement responsable dans sa sexualité;

(.) Je fais une synthèse partielle. 

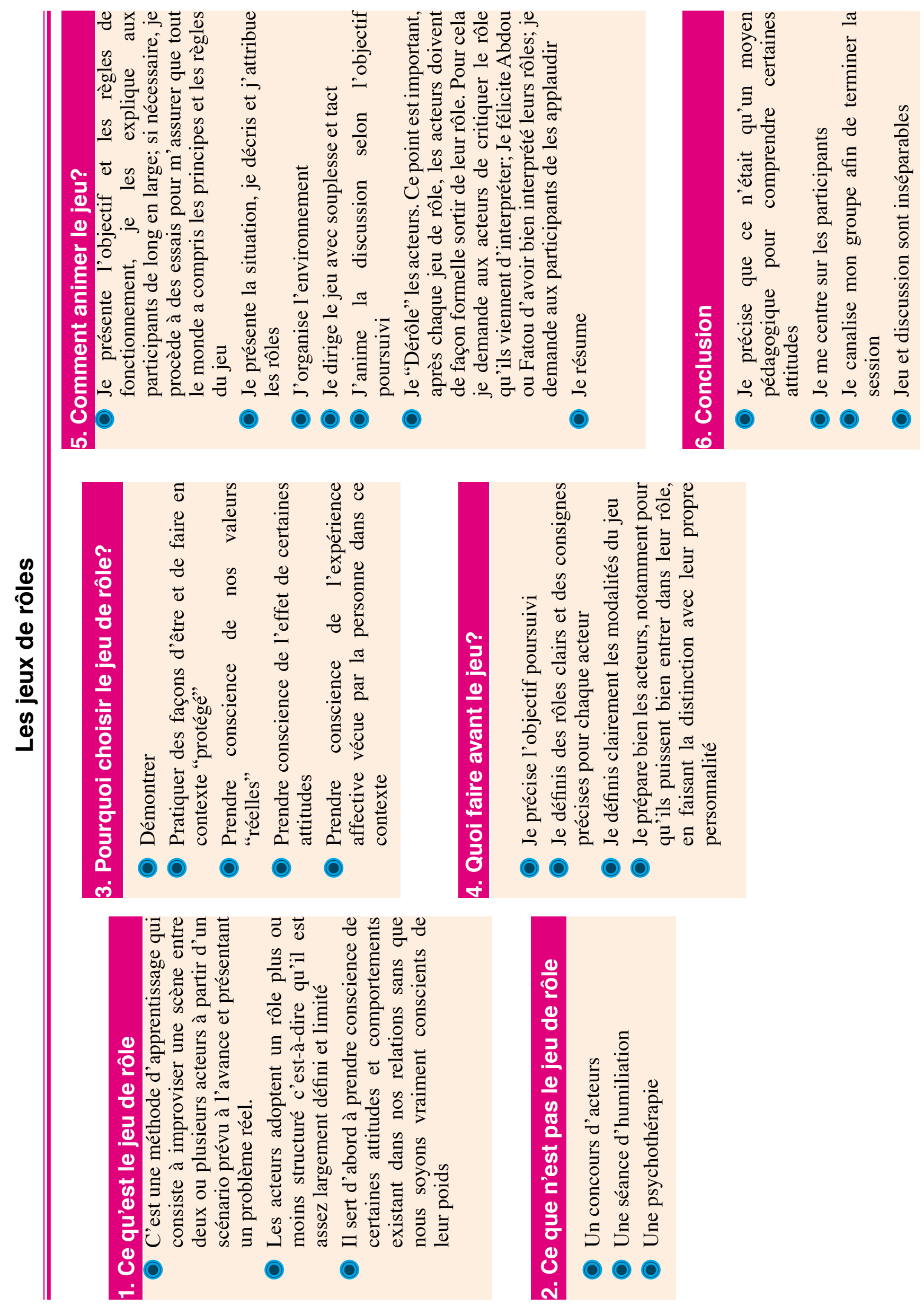


\section{Les jeux "mythes et réalités", "d'accord-pas d'accord"}

Ces jeux sont justement l'occasion de faire émerger et discuter certaines fausses représentations.

C'est l'occasion de défaire les rumeurs et les clichés.

Après les réponses des participants, je fais une synthèse.

\section{L'évaluation}

Souvent, à la fin d'une séance, alors que l'on croit avoir clairement abordé chacune des questions discutées et fait passer des messages positifs et clairs, il arrive que les participants aient compris des choses vraiment différentes (pour différentes raisons liées aux obstacles à la communication mentionnées plus bas);

(1) me faut toujours finir la séance avec un petit moment d'évaluation;

(1) Il ne faut pas donner l'impression aux participants que je les juge. L'évaluation dont on parle ici n'est pas un examen;

(1) Il ne s'agit donc pas d'un contrôle scolaire, ni d'un exercice écrit;

( Il me faut toujours vérifier que l'essentiel des informations et des messages positifs ont été bien retenus par les participants;

(2) Par des questions-réponses, ou en demandant à l'un des participants de rappeler les principaux points discutés lors de la séance et les grandes leçons qu'on en a tirées pour une vie responsable, je peux facilement voir dans quelle mesure les objectifs du module sont atteints ou non;

(1) Cela me permet, lorsque c'est nécessaire, de rectifier certaines informations mal comprises ou de mettre l'accent sur une conduite à tenir jugée importante.

\section{Les points à retenir}

(2) Au cours des discussions, qui suivent les différents jeux "mythes et réalités", "jeux de rôle", etc., il est possible que certains participants défendent avec conviction des positions qui ne sont pas basées sur une bonne information;

( ) Sans pour autant montrer que je détiens la vérité, je dois aider le groupe à retenir de la séance les éléments les plus positifs pour gérer le présent et l'avenir avec le maximum de chances pour réussir dans la vie;

( Les points à retenir sont importants pour cela et je dois bien les avoir à l'esprit tout au long de la séance;

(.) Il ne faut pas que je passe là-dessus trop rapidement, mais au contraire il faut que j'essaie le plus habilement possible, de montrer comment tout ce qu'on a vu au cours de la séance pousse à retenir ces points-là, pour un adolescent qui veut sauvegarder ses chances de réussite.

\section{Mieux communiquer avec les adolescents}

La résolution d'un problème en équipe est l'une des valeurs réelles d'une approche participative en éducation/ formation.

J'évite que la communication soit unilatérale; je n'arriverai à rien si je m'approprie la parole et la connaissance et dis aux autres ce qu'il faut penser ou faire. C'est l'une des raisons pour lesquelles "Grandir en harmonie" utilise une approche interactive et participative. 


\begin{tabular}{|l|l|}
\hline Je dois & Je ne dois pas \\
\hline Encourager la discussion & Menacer et imposer mon opinion \\
\hline Ecouter sans juger & Juger ou critiquer \\
\hline Respecter l'opinion des autres & Ignorer les opinions des autres \\
\hline Donner des messages directs, clairs et accessibles & Donner des messages confus \\
\hline $\begin{array}{l}\text { Essayer de montrer des situations de gagnant. } \\
\text { M'excuser lorsque j'ai commis une erreur. Utiliser les } \\
\text { bonnes manières. Respecter les participants. }\end{array}$ & $\begin{array}{l}\text { Blâmer la personne et montrer des situations } \\
\text { d'échec, de perte } \\
\text { Eviter les appréciations superficielles }\end{array}$ \\
\hline $\begin{array}{l}\text { Dissocier l'appréciation du problème sans accuser la } \\
\text { personne }\end{array}$ & Attaquer la personne plutôt que le problème \\
\hline Etre confiant et maintenir le contact des yeux & Avoir peur de regarder la personne dans les yeux \\
\hline $\begin{array}{l}\text { Utiliser un corps et un langage non menaçant } \\
\text { Je dois encourager les participants lorsqu'ils s'expriment } \\
\text { en acquiesçant de la tête }\end{array}$ & Utiliser un langage corporel désapprobateur \\
\hline
\end{tabular}

La première étape pour éveiller l'intérêt de quelqu'un c'est de ne pas utiliser un langage gestuel ou verbal menaçant. Je dois inspirer la confiance, montrer que j'ai confiance en moi et ne pas fuir les regards.

Quelques techniques de communication pour faciliter la conduite de mes activités de Communication pour un Changement de Comportement (CCC).

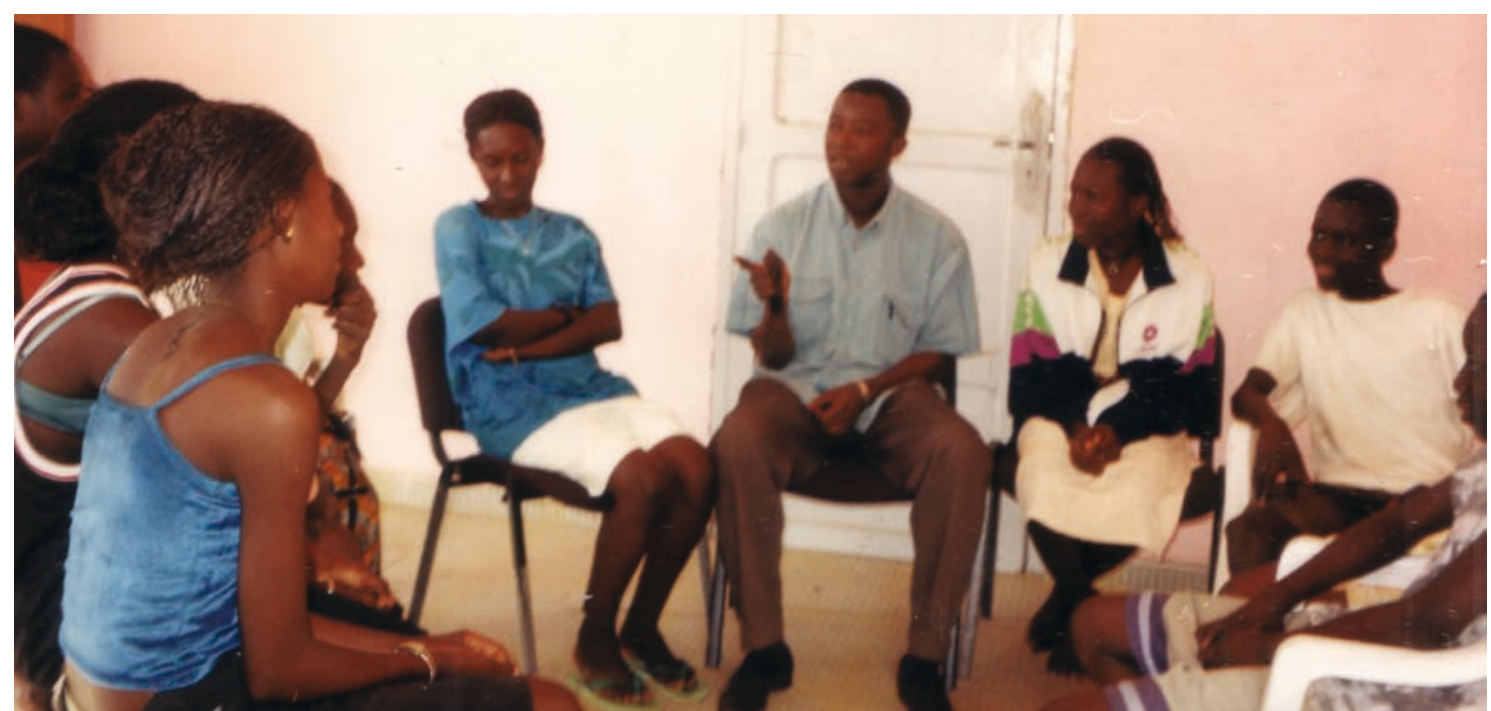




\begin{tabular}{|c|c|c|}
\hline Au niveau & La communication passe bien... & \begin{tabular}{|l|} 
Il y a obstacle à la \\
communication s'il y a...
\end{tabular} \\
\hline \multirow{10}{*}{ de mon attitude } & si je suis: & non-maîtrise du sujet \\
\hline & motivé & langage non adapté \\
\hline & correctement habillé & ignorance \\
\hline & ponctuel & impatience \\
\hline & disponible & distraction \\
\hline & clair dans ma diction & non respect du public-cible \\
\hline & courtois & voix inaudible \\
\hline & crédible & manque de concision \\
\hline & \multirow[t]{2}{*}{ pertinent } & volubileté \\
\hline & & manque d'assurance \\
\hline \multirow{6}{*}{ du message } & si le message est: & un message: \\
\hline & clair et concis & inadapté \\
\hline & précis & complexe \\
\hline & attrayant & confus \\
\hline & \multirow[t]{2}{*}{ adapté à l'auditoire } & long \\
\hline & & difficile à comprendre \\
\hline \multirow{6}{*}{ du bénéficiaire } & si le bénéficiaire est: & un bénéficiaire: \\
\hline & intéressé & distrait ou fatigué \\
\hline & attentif & désintéressé \\
\hline & disponible & indisponible \\
\hline & réceptif & \\
\hline & motivé & \\
\hline \multirow{6}{*}{$\begin{array}{l}\text { du canal (parole, image, films, } \\
\text { etc.) }\end{array}$} & si le canal est: & un canal: \\
\hline & en bon état & défaillant \\
\hline & accessible & inadapté \\
\hline & efficace & en mauvais état \\
\hline & adapté & non approprié \\
\hline & disponible & \\
\hline \multirow{6}{*}{ de la réaction (ou feed-back) } & si la réaction est: & une réaction: \\
\hline & claire & de silence \\
\hline & immédiate & non immédiate \\
\hline & pertinente & non pertinente \\
\hline & \multirow[t]{2}{*}{ positive et constructive } & négative \\
\hline & & destructrice \\
\hline \multirow{6}{*}{ de l'environnement } & si l'environnement est: & un environnement: \\
\hline & agréable & inadapté \\
\hline & sécurisant & trop chaud \\
\hline & adapté & trop froid \\
\hline & \multirow[t]{2}{*}{ propice à un échange franc et fructueux } & insécurisant \\
\hline & & bruyant \\
\hline
\end{tabular}

